\title{
Produção de juvenis do linguado Paralichthys orbignyanus: efeito da duração do período de co-alimentação durante o desmame
}

\author{
Production of Brazilian flounder Paralichthys orbignyanus juveniles: the effect of co-feeding period \\ during the weaning
}

\author{
Andréa Ferretto da Rocha' Cristina Vaz Avelar de Carvalho' Luís André Sampaio ${ }^{1 *}$
}

\section{RESUMO}

O desmame, ou seja, a substituição do alimento vivo por alimento inerte é uma etapa crítica para a produção de alevinos de peixes marinhos. Este trabalho teve por objetivo avaliar o efeito do período de co-alimentação (alimento vivo e inerte) sobre a sobrevivência, o crescimento e os custos de produção de juvenis do linguado Paralichthys orbignyanus. Durante o período de co-alimentação, os juvenis (idade inicial 32 dias) receberam Artemia enriquecida juntamente com ração. Foram testados três períodos de co-alimentação: 15, 20 e 25 dias, mais um grupo controle alimentado com Artemia enriquecida. Ao final do experimento (76 dias), os juvenis alimentados exclusivamente com Artemia enriquecida apresentaram sobrevivência (82\%), peso $(480 \pm 157 \mathrm{mg}) e$ comprimento $(35,5 \pm 5,0 \mathrm{~mm})$ significativamente maiores $(P<0,05)$ que os juvenis alimentados com ração. Não houve diferença significativa $(P>0,05)$ no crescimento entre os juvenis alimentados com ração nos diferentes períodos, entretanto, um período maior de co-alimentação favoreceu a sobrevivência. Os resultados obtidos demonstram que os linguados alimentados exclusivamente com Artemia apresentam melhor performance do que aqueles alimentados com ração. Entretanto, o custo de produção quando a Artemia é substituída por ração aos 58 dias de vida é menor do que quando o desmame é realizado mais cedo ou quando os alevinos são alimentados apenas com Artemia.

Palavras-chave: co-alimentação, desmame, Paralichthys orbignyanus, dieta artificial.

\section{ABSTRACT}

Weaning is a critical period for production of marine fish juveniles. The objective of this research was to evaluate the effect of co-feeding on survival, growth, and cost of production after the weaning of juveniles of Brazilian flounder Paralichthys orbignyanus. Juvenile flounder (32 days after hatching) were fed on HUFA enriched Artemia and subsequently three periods of co-feeding with dry diets (15, 20, and 25 days) were tested, a control group, where flounder were fed exclusively on Artemia was also followed until the end of the experiment. Juveniles fed on Artemia showed significantly higher $(P<0.05)$ survival (82\%), weight $(480 \pm 157 \mathrm{mg})$ and total length $(35.5 \pm 5.0 \mathrm{~mm})$ than flounder weaned into dry diets, independent of the co-feeding period. Growth was similar $(P>0.05)$ for juveniles fed on dry diets, but a higher survival rate was registered when the co-feeding period was longer. The results obtained demonstrate that flounder fed exclusively on Artemia have a better performance than those weaned into dry diets. However, unitary cost of production is reduced when juveniles are weaned 58 days after hatching.

Key words: co-feeding, weaning, Paralichthys orbignyanus, artificial diets.

\section{INTRODUÇÃO}

O linguado Paralichthys orbignyanus é uma das espécies consideradas para desenvolvimento da piscicultura marinha no Brasil (SAMPAIO et al., 2001; SAMPAIO et al., 2007). No entanto, assim como sucede com a maioria das espécies cultivadas, os juvenis de $\boldsymbol{P}$. orbignyanus devem ser capazes de aceitar uma dieta formulada, visto que a utilização do alimento vivo por um período muito extenso pode criar barreiras econômicas significativas para uma produção comercial (HAMLIN \& KLING, 2001).

Na criação de juvenis de peixes marinhos, ainda é necessário o fornecimento de presas vivas durante a primeira alimentação, principalmente rotíferos (Brachionus plicatilis) e Artemia (CAHU \&

IDepartamento de Oceanografia, Fundação Universidade Federal do Rio Grande (FURG), 96201-900, Rio Grande, RS, Brasil. Email: sampaio@mikrus.com.br.*Autor para correspondência. 
ZAMBONINO INFANTE, 2001). Entretanto, é necessário esforço em termos de mão-de-obra, tempo e energia para a produção destas duas espécies de alimento vivo, representando uma despesa que pode alcançar $80 \%$ do custo total de produção (PERSON LE RUYET et al., 1993). Para a maioria das espécies de peixes marinhos pesquisadas, os resultados encontrados nos estudos que avaliam a substituição do alimento vivo pelo alimento inerte, conhecida também como "desmame", mostram uma menor eficiência das dietas formuladas em comparação com o alimento vivo quando utilizadas como única fonte alimentar desde a primeira alimentação (HOLT 1993, KOLKOVSKI et al., 1997). Quando se procede à transição alimentar de uma forma gradativa, em que o alimento inerte é oferecido em combinação com o alimento vivo, denominada estratégia de co-alimentação, podem ser obtidas taxas de crescimento similares ou melhores às alcançadas com o fornecimento exclusivo de Artemia (PERSON LERUYET et al., 1993).

As vantagens obtidas com o uso da estratégia de co-alimentação na realização do desmame, possibilitam uma redução na quantidade de alimento vivo utilizado e diminuem, conseqüentemente, os custos de produção (CALLAN et al., 2003). Este regime alimentar pode auxiliar as larvas e os juvenis a aceitarem o alimento inerte e, de uma forma geral, isso pode resolver o problema do fornecimento inadequado de nutrientes, devido à maior diversidade de alimento disponível (ROSELUND et al., 1997). Resultados positivos em sobrevivência e crescimento foram observados no desmame de Hippoglossus hippoglossus, assim como em Dicentrarchus labrax e Sparus aurata. Esses estudos concluíram que o desmame após 10 dias de co-alimentação seguido de sete dias de redução da densidade de Artemia (ROSELUND et al., 1997). HART \& PURSER (1996), utilizando a estratégia de co-alimentação de 10 dias, alcançaram sobrevivência superior no desmame do linguado Rhombosolea tapirina aos 23 dias após a eclosão. Entretanto, muitos fatores estão envolvidos no sucesso da substituição do alimento vivo pelo alimento inerte, tais como o desenvolvimento do sistema digestório, o comportamento alimentar, a duração do período de transição alimentar e as propriedades da dieta (PERSON LE RUYET et al., 1993), que devem ser levados em consideração antes da escolha de um protocolo adequado de alimentação.

Este estudo foi realizado para determinar o tempo mínimo necessário de co-alimentação antes de se completar o desmame de juvenis de $\boldsymbol{P}$. orbignyanus, comparando quatro estratégias alimentares em termos de sobrevivência, crescimento e custos de produção.

\section{MATERIAL E MÉTODOS}

O experimento foi realizado no Laboratório de Maricultura, do Departamento de Oceanografia, da Fundação Universidade Federal do Rio Grande (FURG). Os juvenis de $\boldsymbol{P}$ orbignyanus foram obtidos a partir de reprodução em laboratório de linguados capturados na natureza. Os reprodutores foram induzidos hormonalmente à desova em laboratório utilizando-se extrato de hipófise de carpa na dose única de $3 \mathrm{mg} \mathrm{kg}^{-1}$.

A primeira alimentação das larvas foi iniciada com o rotífero B. plicatilis a uma densidade de 20 indivíduos $\mathrm{mL}^{-1}$. Os rotíferos foram alimentados com a microalga Nannochloropsis oculata a uma densidade de $0,5 \times 10^{6}$ células $\mathrm{ml}^{-1}$ nos tanques de larvicultura e Culture Selco 3000 (Inve). Aos nove dias após eclosão (DAE) foi iniciado o fornecimento de Artemia enriquecida (1-5 Artemia ml ${ }^{-1}$ ) juntamente com o rotífero até completarem 32 dias de vida. Os cistos de Artemia (Inve) eram colocados para eclodir durante 24 horas e os náuplios enriquecidos durante 24 horas com DHA Selco (Inve). Após esse período, a Artemia enriquecida era coletada e fornecida às larvas e aos juvenis durante o dia, permanecendo refrigeradas e com aeração.

Ao completarem 32 dias de vida, os juvenis foram medidos (comprimento total 9,5 $\pm 1,2 \mathrm{~mm}, \mathrm{n}=35$ ), contados e transferidos para tanques de fibra de vidro de $50 \mathrm{~L}$, com paredes pretas e fundo branco, a uma densidade de 3 indivíduos $\mathrm{L}^{-1}$, ou 150 juvenis em cada tanque. Os tanques permaneceram imersos em banho termostatizado com temperatura média de $23,8 \pm 0,2^{\circ} \mathrm{C}$ e água do mar (salinidade entre 30 e 34\%o), em sistema de fluxo-contínuo, com uma taxa de renovação de aproximadamente 10 litros $\mathrm{h}^{-1}$. O experimento teve duração de 44 dias, quando os linguados completaram 76 dias de vida

A concentração de amônia total foi medida segundo metodologia da UNESCO (1983) e nunca excedeu $0,3 \mathrm{mg} \mathrm{L}^{-1} \mathrm{~N}-\mathrm{NH}_{3}$, enquanto o $\mathrm{pH}$ esteve entre 7,8 e 8,1 . Os tanques eram sifonados diariamente para a retirada do material depositado no fundo. A água foi filtrada por um sistema de filtros de cartucho $(1 \mu \mathrm{m}) \mathrm{e}$ ultravioleta. $\mathrm{O}$ fotoperíodo foi alterado para 18 horas de luz e seis horas de escuro, com intensidade luminosa de aproximadamente 200 lux na superfície da água.

Foram avaliados quatro tratamentos com três repetições, sendo três períodos de co-alimentação e um grupo controle, em que as larvas foram alimentadas exclusivamente com Artemia. O protocolo experimental pode ser observado na figura 1 , sendo descrito como:

ART: juvenis alimentados exclusivamente com Artemia ao longo do experimento. 


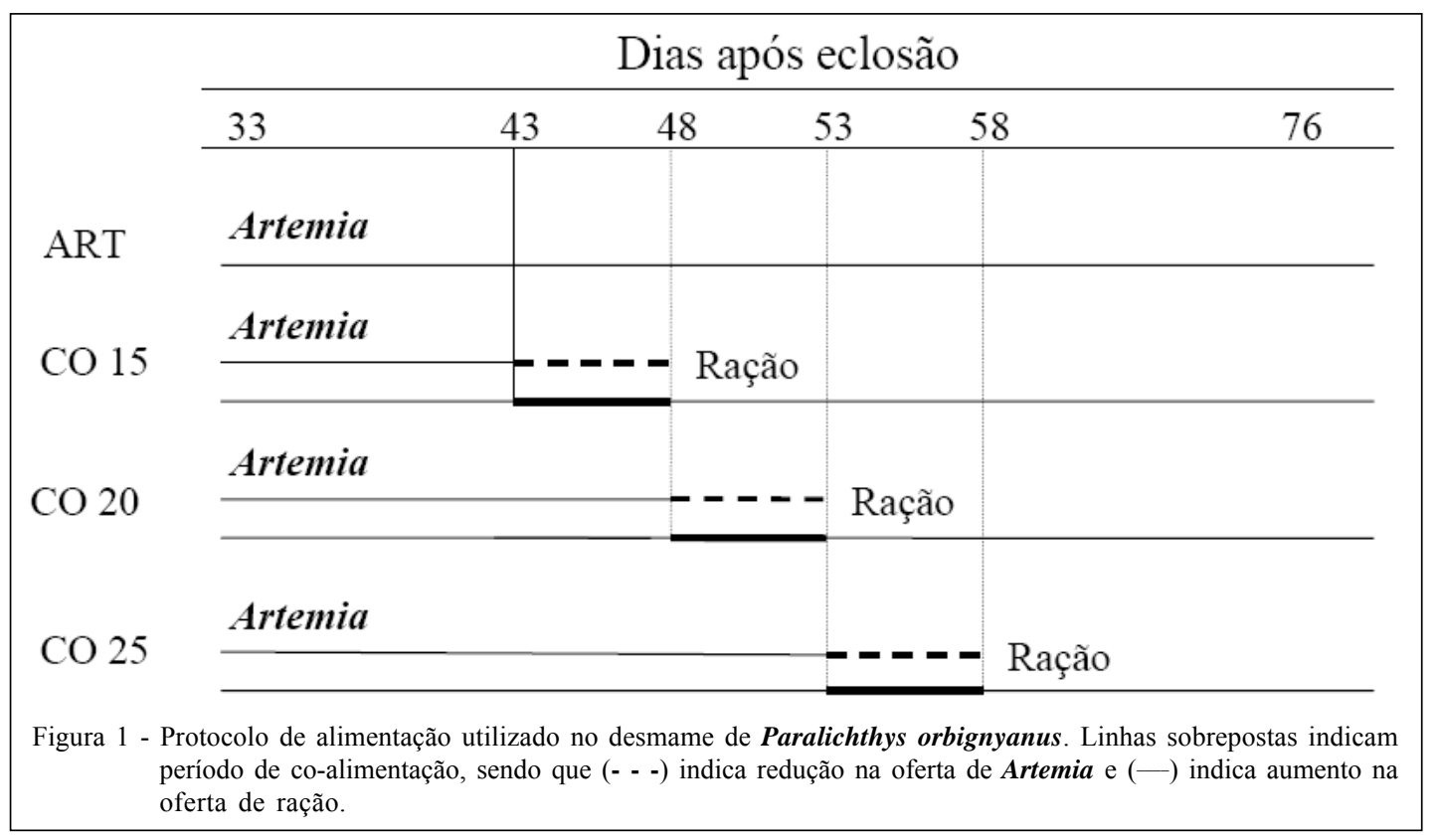

CO-15: ao completarem 33 dias de vida, os juvenis deste grupo foram co-alimentados (Artemia e ração) durante 15 dias. Nos últimos cinco dias, foi iniciada a redução gradual da oferta de Artemia concomitante ao aumento da oferta de ração, efetivando-se o desmame aos 48 DAE.

CO-20: ao completarem 33 dias de vida, os juvenis deste grupo foram co-alimentados (Artemia e ração) durante 20 dias. Nos últimos cinco dias, foi iniciada a redução gradual da oferta de Artemia concomitante ao aumento da oferta de ração, efetivando-se o desmame aos 53 DAE.

CO-25: ao completarem 33 dias de vida, os juvenis deste grupo foram co-alimentados (Artemia e ração) durante 25 dias. Nos últimos cinco dias, foi iniciada a redução gradual da oferta de Artemia concomitante ao aumento da oferta de ração, efetivando-se o desmame aos 58 DAE.

No início do experimento, foi utilizada a ração comercial Proton 2 (Inve), com 58\% de proteína, $14 \%$ de lipídio, $9 \%$ de cinzas e tamanho de partícula entre 150-300 $\mu \mathrm{m}$ de diâmetro, juntamente com a ração Lansy NRD (Inve), com $60 \%$ de proteína, $14,5 \%$ de lipídio, $11,5 \%$ de cinzas e tamanho de partícula entre

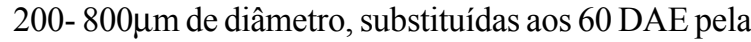
ração Epac Alfa (Inve), com $56 \%$ de proteína, 15\% de lipídio, $12 \%$ de cinzas e tamanho de partícula entre 1200-2000 $\mu \mathrm{m}$ de diâmetro. Também foi oferecida Artemia enriquecida com DHA Selco (Inve) para todos os tratamentos durante os períodos de co-alimentação.

A oferta do alimento vivo foi realizada três vezes ao dia (manhã, tarde e noite), enquanto o alimento inerte foi oferecido, no mínimo, duas vezes pela manhã, três vezes pela tarde e duas vezes pela noite, sempre com sobras.

Para análise do crescimento, os juvenis de linguado foram pesados em balança de precisão e medidos sob microscópio estereoscópico ao final do experimento. Para estimar a sobrevivência (S), os juvenis foram contados no final do experimento (Nf). O cáclculo foi efetuado com a fórmula: $S=(\mathrm{Ni}-\mathrm{N} f) / \mathrm{Ni}$ x 100, em que Ni é o número inicial de juvenis de linguado. A taxa de crescimento específico (TCE) foi calculada a partir da equação: $\mathrm{TCE}=(\ln \mathrm{C} f-\ln \mathrm{Ci}) / \mathrm{T} \times 100$, sendo que: $\mathrm{C}$ fé o comprimento final, $\mathrm{C} i$ é o comprimento inicial e $\mathrm{T}$ é a duração do período experimental em dias. $\mathrm{O}$ cálculo dos custos com alimentação foi baseado no consumo aparente de Artemia enriquecida e ração durante o experimento. Foi considerada a eclosão de 150.000 náuplios de Artemia/grama de cisto.

A normalidade dos dados e a homocedasticidade da variância foram testadas respectivamente com os testes de KolmogorovSmirnov e Levene. Os resultados de sobrevivência, taxa de crescimento específico, comprimento e peso foram analisados com ANOVA (uma via), seguida do teste de Tukey quando encontrada diferença significativa entre os tratamentos. Os dados percentuais foram transformados (arco seno) antes de serem submetidos à ANOVA. Todas as análises foram realizadas com um nível de significância de $95 \%$ (SOKAL \& ROHLF 1995), utilizando o programa estatístico Statistica 6.0.

\section{RESULTADOS E DISCUSSÃO}

O grupo alimentado exclusivamente com Artemia apresentou sobrevivência significativamente 
maior $(\mathrm{P}<0,05)$ que os grupos que passaram pelo processo de transição alimentar. A sobrevivência do tratamento $\mathrm{CO} 15$ foi significativamente menor do que CO $25(\mathrm{P}<0,05)($ Tabela 1$)$.

A baixa sobrevivência verificada nos tratamentos que completaram o desmame pode ser atribuída ao fato de os juvenis não se adaptarem à dieta inerte e morrerem por inanição após a retirada completa do alimento vivo. As larvas de peixes marinhos não dispõem de enzimas digestivas suficientes para digerir alimento artificial (CAHU \& ZAMBONINO INFANTE, 2001), portanto, também é possível que apesar de terem ingerido a ração, ela não tenha sido digerida e/ou assimilada. LEE \& LITVAK (1996) obtiveram alta sobrevivência no desmame de juvenis de Pleuronectes americanus utilizando um protocolo de desmame gradual de sete dias. HART \& PURSER (1996) observaram que larvas do linguado Rhombosolea tapirina alcançavam sobrevivência similar em diferentes períodos de co-alimentação.

A taxa de crescimento específico em comprimento foi significativamente maior para os juvenis alimentados exclusivamente com Artemia $(\mathrm{P}<0,05)$. Entretanto, não foi observada diferença significativa $(\mathrm{P}>0,05)$ para os linguados alimentados com ração, independente do período de co-alimentação utilizado. Os mesmos resultados foram observados para o peso úmido e o comprimento total ao final do experimento (Tabela 1). O aumento do período de coalimentação pode proporcionar uma melhor performance, como observado por HART \& PURSER (1996) em Rhombosolea tapirina, em que o tempo para concluir o desmame apresentou efeito significativo sobre o crescimento, mostrando que períodos mais longos (10-20 dias) são mais eficientes que períodos menores (cinco dias).

Para que o sistema de co-alimentação seja bem-sucedido, é importante que a larva se alimente da ração enquanto o alimento vivo ainda é oferecido (ROSELUND et al., 1997). KOLKOVSKI et al., (1997) também observaram que larvas de pargo Sparus aurata previamente alimentadas com presas vivas selecionam preferencialmente o alimento vivo durante a coalimentação, o que pode refletir em um comportamento de pré-condicionamento em favor do alimento vivo. Entretanto, APPELBAUM (1985) observou que larvas de Solea solea aceitaram mais facilmente partículas inertes nos primeiros estádios de desenvolvimento do que em estádios mais avançados. Também CAÑAVATE \& FERNÁNDEZ-DÍAZ (1999) verificaram que a oferta de Artemia e ração na proporção de 1:1 desde a primeira alimentação faz com que um maior número de larvas de Solea senegalensis aceite a ração mais cedo. Há sugestões de um regime de co-alimentação precoce favorece a aceitação do alimento inerte, acelerando o processo de substituição do alimento vivo (ROSELUND et al., 1997; CALLAN et al., 2003), facilitando o processo de digestão e assimilação de nutrientes(HOLT, 1993; PERSONLERUYET et al., 1993) e garantindo uma significativa economia no custo de produção com alimento vivo (KOLKOVSKI et al., 1997).

As observações comportamentais também devem ser levadas em consideração, pois podem ajudar a explicar os dados de crescimento e sobrevivência. BENETTI (1997) sugere estocar juvenis de Paralichthys woolmani em baixas densidades (5 indivíduos $\mathrm{L}^{-1}$ ) e fazer um constante gradeamento para reduzir o canibalismo. Neste trabalho foram observadas situações de canibalismo entre os juvenis de $\boldsymbol{P}$. orbignyanus principalmente após o desmame. $\mathrm{O}$ gradeamento não foi realizado, entretanto, a densidade de estocagem utilizada ( 3 indivíduos $\mathrm{L}^{-1}$ ) é a usualmente empregada em estudos com outras espécies de linguado (LEE \& LITVAK, 1996).

A análise dos custos com alimentação durante o experimento mostrou que os gastos com Artemia foram muito superiores aos gastos com ração. Isso tem reflexo nos custos de produção, pois embora a utilização da Artemia tenha favorecido a sobrevivência e o crescimento, um período de coalimentação de 25 dias reduziu os custos finais (Tabela 2).

$\mathrm{O}$ alimento vivo pode representar $80 \%$ do custo total em 45 dias de produção de robalo europeu Dicentrarchus labrax, sendo que o uso de Artemia

Tabela 1 - Performance de juvenis de linguado Paralichthys orbignyanus sob diferentes diferentes regimes alimentares: co-alimentados com Artemia e ração durante 15, 20 e 25 dias (CO 15, CO 20 e CO 25) ou exclusivamente com Artemia

\begin{tabular}{|c|c|c|c|c|}
\hline Parâmetro analisado & Artemia & $\mathrm{CO} 25$ & CO 20 & CO 15 \\
\hline Sobrevivência & $82 \pm 3^{\mathrm{a}}$ & $23 \pm 3^{b}$ & $13 \pm 5^{\text {bc }}$ & $9 \pm 6^{\mathrm{c}}$ \\
\hline Peso úmido & $480 \pm 157^{\mathrm{a}}$ & $192 \pm 161^{b}$ & $243 \pm 207^{b}$ & $184 \pm 10^{\mathrm{b}}$ \\
\hline Comprimento total & $35,5 \pm 5^{\mathrm{a}}$ & $26,5 \pm 5^{\mathrm{b}}$ & $27,4 \pm 6^{\mathrm{b}}$ & $24,6 \pm 6^{\mathrm{b}}$ \\
\hline TCE - comprimento & $3,0 \pm 0,32^{\mathrm{a}}$ & $2,3 \pm 0,46^{\mathrm{b}}$ & $2,4 \pm 0,47^{\mathrm{b}}$ & $2,1 \pm 0,55^{\mathrm{b}}$ \\
\hline
\end{tabular}

* Letras diferentes indicam diferença estatística $(\mathrm{P}<0,05)$ após teste de Tukey. 
Tabela 2 - Custos de produção de juvenis do linguado Paralichthys orbignyanus com Artemia e ração.

\begin{tabular}{lcccc}
\hline Tratamento & Artemia $^{1}$ & Ração $^{2}$ & DHA $^{3}$ & Total $^{2}$ \\
\hline Artemia & 41 & --- & 32 & 173 \\
CO 15 & 88 & 61 & 20 & 169 \\
CO 20 & 104 & 41 & 23 & 168 \\
CO 25 & 95 & 22 & 21 & 138 \\
\hline
\end{tabular}

Os valores são apresentados em dólar americano.

${ }^{1}($ Inve $)=\mathrm{US} \$ 80,00 \mathrm{~kg}^{-1} ;{ }^{2}$ (Inve) $=\mathrm{US} \$ 15,00 \mathrm{~kg}^{-1} ;{ }^{3}$ (Inve $)=\mathrm{US} \$ 48,00 \mathrm{~L}^{-1}$.

representa $80 \%$ do total de despesas com alimento vivo, que incluem também os gastos com a produção de microalgas e rotíferos (PERSON LE RUYET et al., 1993). CALLAN et al. (2003) obtiveram resultados positivos em sobrevivência e crescimento do bacalhau Gadus morhua utilizando um sistema de co-alimentação que reduziu cerca de $75 \%$ a quantidade de Artemia. EHRLICH et al. (1989) conseguiram reduzir o custo de produção de Micropterus dolomieui pela metade realizando o desmame mais cedo.

\section{CONCLUSÃO}

Um período de co-alimentação de 25 dias no desmame de $\boldsymbol{P}$. orbignyanus parece ser a melhor estratégia, visto que a sobrevivência dos juvenis foi favorecida por este regime alimentar e que o custo de produção foi menor. Entretanto, ainda não foi possível estabelecer um protocolo ideal de substituição do alimento vivo pelo alimento inerte na criação de juvenis de $\boldsymbol{P}$. orbignyanus.

\section{AGRADECIMENTOS}

Andréa Ferretto da Rocha foi aluna do Programa de Pós-graduação em Aqüicultura da FURG, sendo bolsista de mestrado do CNPq. Cristina Vaz Avelar de Carvalho é aluna do Programa de Pós-graduação em Aqüicultura da FURG. Luís André Sampaio é Bolsista de Produtividade em Pesquisa do CNPq. Este projeto foi financiado com recursos do CT-Agronegócio/ MCT/SEAP-PR/CNPq (Processo 504028/2003-0) e FAPERGS (Processo 04/0501-1).

\section{REFERÊNCIAS}

APPELBAUM, S. Rearing of Dove sole Solea solea L., through its larval stages using artificial diets. Aquaculture, v.49, p.209$221,1985$.

BENETTI, D.D. Spawning and larval husbandry of flounder (Paralichthys woolmani) and Pacific yellowtail (Seriola mazatlana), new candidate species for aquaculture. Aquaculture, v.155, p.307-318, 1997.

CAHU, C.; ZAMBONINO INFANTE, J. Substitution of live food by formulated diets in marine fish larvae. Aquaculture, v.200, p.161-180, 2001.
CALlan, C. et al. Reducing Artemia use in the culture of Atlantic cod (Gadus morhua). Aquaculture, v.219, p.585595, 2003.

CAÑAVATE, J.P.; FERNÁNDEZ-DÍAZ, C. Influence of cofeeding larvae with live and inert diets on weaning the sole Solea senegalensis onto commercial dry feeds. Aquaculture, v.174, p.255-263, 1999.

EHRLICH, K.F. et al. Growth and survival of larval and postlarval smallmouth bass fed a commercially prepared dry feed and/or Artemia nauplii. Journal of the World Aquaculture Society, v.20, p.1-6, 1989.

HAMLIN, H.J.; KLING, L.J. The culture and early weaning of larval haddock (Melanogrammus aeglefinus) using a microparticulate diet. Aquaculture, v.201, p.61-72, 2001.

HART, P.R.; PURSER, G.J. Weaning of hatchery-reared greenback flounder (Rhombosolea tapirina Güinther) from live to artificial diets: Effects of age and duration of the changeover period. Aquaculture, v.145, p.171-181, 1996.

HOLT, J.G. Feeding larval red drum on microparticulate diets in a closed recirculating water system. Journal of the World Aquaculture Society, v.24, p.225-230, 1993.

KOLKOVSKI, S. et al. The mode of action of Artemia in enhancing utilization of microdiet by gilthead seabream Sparus aurata larvae. Aquaculture, v.155, p.193-205, 1997.

LEE, G.W.Y.; LITVAK, M.K. Weaning of metamorphosed winter flounder (Pleuronectes americanus) reared in the laboratory: comparison of two commercial artificial diets on growth, survival and conversion efficiency. Aquaculture, v. 144, p. 251-263, 1996.

PERSON LE RUYET, J. et al. Marine fish larvae feeding: formulated diets or live prey? Journal of the World Aquaculture Society, v.24, p.211-224, 1993.

ROSELUND, G. et al. Co-feeding marine fish larvae with inert and live diets. Aquaculture, v.155, p.183-191, 1997.

SAMPAIO, L.A. et al. Growth of Brazilian flounder Paralichthys orbignyanus cultured in different salinities. Journal of Applied Aquaculture, v.11, p.67-75, 2001.

SAMPAIO, L.A. et al. Effects of salinity on Brazilian flounder Paralichthys orbignyanus from fertilization to juvenile settlement. Aquaculture, v.262, p.340-346, 2007.

SOKAL, R.R; RHOLF, J. Biometry: the principles and practice of statistics in biological research. 3.ed. New York: State University of New York, 1995. 887p.

UNESCO. Chemical methods for use in marine environmental monitoring. Paris: Intergovernmental Oceanographic Commission, 1983. 53p. (Manual and Guides 12). 